\title{
Application of CNN-LSTM in Gradual Changing Fault Diagnosis of Rod Pumping System
}

\author{
Yanfeng He $\mathbb{D}^{1},{ }^{1}$ Yali Liu $\left(\mathbb{D},{ }^{1}\right.$ Shuai Shao ${ }^{D},{ }^{1}$ Xuhang Zhao $\mathbb{D},{ }^{1}$ Guojun Liu $\mathbb{D},{ }^{2}$ \\ Xiangji Kong $\mathbb{C}^{2},{ }^{2}$ and Lu Liu $\mathbb{\circledR}^{1}$ \\ ${ }^{1}$ School of Petroleum Engineering, Changzhou University, Changzhou, Jiangsu 213164, China \\ ${ }^{2}$ China National Oil and Gas Exploration and Development Company Ltd., Beijing 100034, China \\ Correspondence should be addressed to Yanfeng He; heyanfeng@cczu.edu.cn and Yali Liu; 1621905379@qq.com
}

Received 15 May 2019; Revised 14 August 2019; Accepted 5 September 2019; Published 3 November 2019

Academic Editor: Florin Stoican

Copyright (C) 2019 Yanfeng He et al. This is an open access article distributed under the Creative Commons Attribution License, which permits unrestricted use, distribution, and reproduction in any medium, provided the original work is properly cited.

Owing to the importance of rod pumping system fault detection using an indicator diagram, indicator diagram identification has been a challenging task in the computer-vision field. The gradual changing fault is a special type of fault because it is not clearly indicated in the indicator diagram at the onset of its occurrence and can only be identified when an irreversible damage in the well has been caused. In this paper, we proposed a new method that combines the convolutional neural network (CNN) and long short-term memory (LSTM) network to perform a gradual changing fault classification. In particular, we employed CNN to extract the indicator diagram multilevel abstraction features based on its hierarchical structure. We considered the change in the time series of indicator diagrams as a sequence and employed LSTM to perform recognition. Compared with traditional mathematical model diagnosis methods, CNN-LSTM overcame the limitations of the traditional mathematical model theoretical analysis such as unclear assumption conditions and improved the diagnosis accuracy. Finally, 1.3 million sets of well production were set as a training dataset and used to evaluate CNN-LSTM. The results demonstrated the effectiveness of utilizing CNN and LSTM to recognize a gradual changing fault using the indicator diagram and characteristic parameters. The accuracy reached $98.4 \%$, and the loss was less than $0.9 \%$.

\section{Introduction}

Rod pumping systems are used in approximately $94 \%$ of artificially lifted wells. Owing to their extensive application, detailed understanding of the diagnosis and analysis of a rod-pump working state using the indicator diagram is important. Indicator diagram, which is a closed curve, reflects the variation pattern of a suspended point load in a pumping unit with its displacement, which performs vital functions for identifying the rod-pump production state. Ideally, the ground theoretical indicator diagram of wells is an approximate parallelogram, as shown in Figure 1, whose vertical and horizontal coordinates represent the load and displacement, respectively. Section AB represents the loading section in the upward direction, and section $\mathrm{BC}$ represents the upward movement of the sucker rod. Section $\mathrm{CD}$ represents the unloading section in the downward direction, and section DA represents the downward movement of the rod pump.

Currently, three methods are available to analyze the indicator diagram, namely, statistical method [1], knowledge-based fault diagnosis method [2], and artificial neural network (ANN) method. The traditional indicator diagram recognition method suffers from serious insufficiency in that its recognition accuracy is poor and its identification efficiency is low. ANN is the most suitable method for identifying the indicator diagram owing to its self-studying and fault-tolerance qualities. In the field of computer-vision research, the most classic and widely used structure of ANN is the convolutional neural network $(\mathrm{CNN})$, which is a deep feed-forward neural network [3]. With its typical features consisting of concatenating convolution, local connection, weight sharing, and pooling, CNN can effectively reduce the network complexity and the number of training parameters. 


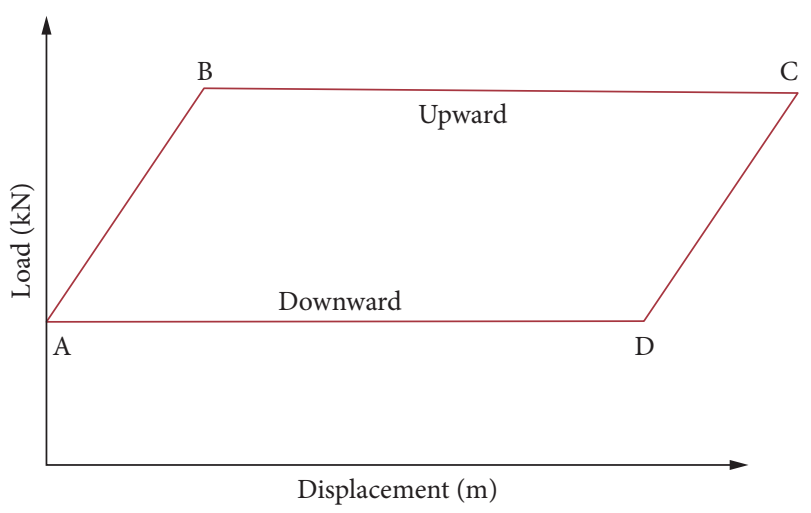

FIgURE 1: Ideal indicator diagram.

Owing to its unique strong robustness and fault-tolerance ability, CNN can be easily trained and optimized. CNN suffers from extreme difficulty in identifying an image that changes over time because of $2 \mathrm{D}$ convolution kernel. In other words, the $\mathrm{CNN}$ channel output after filter calculation only contains the height and width dimensions but no time dimension.

In identifying the indicator diagram using $\mathrm{CNN}$, we find that $\mathrm{CNN}$ can be positively applied in abrupt changing fault identification in which the fault presents obvious mutation results in short time, such as sticking problem, rod parting, and other problems. However, the CNN performance in identifying gradual changing faults [4] is poor. The gradual changing fault is a persistent fault whose features are only obvious to a certain extent until they accumulate for some time, such as casing corrosion, tubing leak, and other faults. We need to note that a gradual changing fault is not clearly indicated in the indicator diagram at the beginning of its occurrence and is identified only when a serious damage is caused to the well at a later stage. In this process, the production capacity of an oil well has already been damaged, and an even irreversible damage might have been caused. To identify a gradual changing fault, we combine the long shortterm memory (LSTM) network with CNN.

LSTM is a type of recurrent neural network (RNN) with a more complex computational unit. LSTM is suitable for handling a variable-length input and easily learns highly nontrivial long-distance dependence because of the forget gate installed in it [5]. Therefore, the CNN-LSTM system can be applied to identify the gradual changing fault by learning the characteristic of the indicator diagram. The specific identification process requires $\mathrm{CNN}$ to extract multiple features from an indicator diagram and input them to LSTM.

LSTM can capture sequential patterns by learning to store or ignore certain information from the sequence inputs [6]. When LSTM takes the CNN output as an input, all previous inputs in the sequence are considered. Therefore, if we treat an indicator diagram as feature sequences, we can intuitively use LSTM to perform fault classification. Furthermore, because $\mathrm{CNN}$ can produce features with different levels of abstraction from an image $[7,8]$ and because the features of the different levels of abstraction can provide complementary information, gradual changing fault identification can be realized.

\section{Parameters}

2.1. Definition of Gradual Changing Fault. The gradual changing fault in a rod pumping system mainly refers to the loss of circulation, casing corrosion, sand production, scale deposition, and pump barrel wear. In the traditional determination method, gradual changing fault is recognized based on experience without employing any systematic or scientific method. The empirical method depends on human judgment, which may cause low accuracy in determining the result and similar-fault-identification error. A determination method called principal component analysis (PCA) is proposed, which can more accurately and reasonably divide a gradual changing fault by measuring the indicator diagram similarity between the normal production and failure processes [9]. PCA is a multivariate statistical technique based on the similarity factor $\left(S_{\mathrm{PCA}}\right)$ of the principal components, which can be used to calculate the principal directions of variability in the data [10].

The process of determining the gradual changing fault using PCA is described as follows: We assume standard working indicator diagram dataset $H$ and new working indicator diagram dataset $S$ that contain the same $n$ variables. PCA analysis is carried out on the $S$ and $H$ datasets, and we assume that the datasets can be constructed using the $K$ principal components contained in the respective PCA models. The corresponding $(n \times k)$ subspaces are denoted by $L$ and $M$, respectively. The PCA similarity factor $\left(S_{\mathrm{PCA}}\right)$ compares these reduced subspaces [11].

$S_{\mathrm{PCA}}$ is defined as

$$
S_{\mathrm{PCA}}^{\lambda}=\frac{\operatorname{trace}\left(\mathbf{R}^{T} \mathbf{T} \mathbf{T}^{T} \mathbf{R}\right)}{\sum_{i=1}^{k} \lambda_{i}^{l} \lambda_{i}^{m}},
$$

where

$$
\begin{aligned}
\mathbf{R} & =\mathbf{L} \Lambda_{l}, \\
\mathbf{T} & =\mathbf{M} \Lambda_{m}, \\
\Lambda_{l} & =\left[\begin{array}{cccc}
\sqrt{\lambda_{1}^{l}} & 0 & 0 & 0 \\
0 & \sqrt{\lambda_{2}^{l}} & 0 & 0 \\
0 & 0 & \ddots & 0 \\
0 & 0 & 0 & \sqrt{\lambda_{i}^{l}}
\end{array}\right], \\
\Lambda_{m} & =\left[\begin{array}{cccc}
\sqrt{\lambda_{1}^{m}} & 0 & 0 & 0 \\
0 & \sqrt{\lambda_{2}^{m}} & 0 & 0 \\
0 & 0 & \ddots & 0 \\
0 & 0 & 0 & \sqrt{\lambda_{i}^{m}}
\end{array}\right] .
\end{aligned}
$$

The more similar subspaces $L$ and $M$ are corresponding to datasets $H$ and $M$, the larger is the value of $S_{\mathrm{PCA}}^{\lambda}$. The PCA similarity factor $\left(S_{\mathrm{PCA}}\right)$ is used to distinguish the fault type. 
According to $S_{\mathrm{PCA}}^{\lambda}$ of 15 typical faults calculated by the SPSS software, the threshold value is set to 0.5 in this study. In other words, when $S_{\mathrm{PCA}}$ between working indicator diagram dataset $S$ and standard working condition indicator diagram dataset $H$ is greater than 0.5 , the new working condition is defined as a gradual changing fault; otherwise, it is defined as an abrupt changing fault.

According to the PCA method, common failures of the rod pumping system are classified into gradual or abrupt changing faults, as listed in Table 1.

After the classification, the type of gradual changing fault is determined, and the characteristic parameters are selected to describe the fault-changing process.

2.2. Characteristic Parameters. Because large variations exist in a single indicator diagram, dealing with a single image in gradual changing fault identification is difficult. If multiple views of successive indicator diagrams can be effectively fused, we can retain information that is useful for the gradual changing fault while searching for variations in images.

The suspended point load of the rod pumping system is the key to ensure normal operation of the rod pump [12]. As an example of scale deposition, the change in the suspended point load during a fault is studied using the method of multiple continuous indicator diagrams, as shown in Figure 2.

Figure 2 shows that section $\mathrm{AB}$ represents the slow scale deposition occurrence with time, point $\mathrm{C}$ denotes the period of fault, and the load sharply increases. Following the occurrence of scale deposition, the load and indicator diagram area gradually increase because the occurrence of scaling creates an additional resistance to the whole stroke or a certain area in the piston. In the upward stroke, the additional resistance increases the rod-pump suspended point load. In the downward stroke, the additional resistance reduces the load, and a vibrational load appears. In summary, the load increases during the upward stroke and decreases during the downward stroke. The phenomenon of scale deposition reflected in the indicator diagram indicates that the load lines in the upward and downward strokes show obvious variation rules.

Hence, load is an indicator of the scale deposition. The development pattern of the gradual changing fault can be identified through the load. The load data are collected from the oilfield and inputted into CNN-LSTM. The load variation in the occurrence process of gradual changing fault identified by CNN-LSTM is shown in Figure 3. The figure shows that the rod pumping system suspended point load exhibits an obvious low periodic change, which reflects the change process of the fault within one cycle. Thus, identifying the gradual changing fault based on the load is feasible.

\section{Approach}

3.1. Framework Overview. In this paper, we propose a scheme to combine CNN and LSTM to perform gradual changing fault identification. The overview of the framework of the proposed method is shown in Figure 4.
TABLE 1: Rod pumping system failure classification.

\begin{tabular}{lcc}
\hline Type & Failure & SPCA \\
\hline & Lost circulation & $>0.5$ \\
& Casing corrosion & $>0.5$ \\
Sradual changing fault & $>0.5$ \\
& Sand production & $>0.5$ \\
& Scale deposition & $>0.5$ \\
& Pump barrel wear & $>0.5$ \\
& Insufficient for liquid & $>0.5$ \\
\hline & Gas impact & $<0.5$ \\
& Sudden touch pump & $<0.5$ \\
& Burst travel valve closed & $<0.5$ \\
Rbrupt changing fault & Rod resistance increases & $<0.5$ \\
& Break off & $<0.5$ \\
& Serious leakage of valve & $<0.5$ \\
& Oscillation production & $<0.5$ \\
& Foreign body inside the pump & $<0.5$ \\
\hline & Changes in stroke &
\end{tabular}

Figure 4 shows that the pipeline of the proposed method mainly consists of three components: indicator diagram classification, CNN-LSTM training, and system application. In the indicator diagram classification stage, from a given dataset, we prepare a set of multiple continuous indicator diagrams for the complete period. Subsequently, in the CNN-LSTM training stage, the image views are fed to CNN for feature extraction, and multiple features from the indicator diagram are extracted using $\mathrm{CNN}$ and inputted to LSTM. To acquire the continuous features of the abstraction, we concatenate the output layer of $\mathrm{CNN}$ and the input layer of LSTM in each image view. Consequently, feature sequences are obtained using $\mathrm{CNN}$, and each feature sequence is processed using the corresponding LSTM. In the system application stage, trained CNN-LSTM is used for gradual changing fault identification, and the CNN-LSTM outputs are classified using the corresponding softmax classifiers, which are combined to determine the category of the input indicator diagram [13].

3.2. Dataset Sources. The dataset is collected from 160 wells in $\mathrm{M}$ oilfield, which is characterized by complex lithology, deep reservoir burial, and poor physical properties. The pipeline control system with a large-scale data management collects the production data every half hour and save them as a CSV file for each month. The data represent the production records of oil wells collected from January 1, 2017 to November 30,2017 . The CSV files are used to generate indicator diagrams. The selected data are processed using MATLAB software, which include the threshold segmentation, imdilate function expansion operations, imerode function corrosion operations, thin function refinement operations, and shrink function shrinkage operations. The CNN-LSTM training dataset is constructed using the processed indicator diagram, and the loads are selected as feature parameters.

3.3. CNN-LSTM Model. Inspired by the application of neural networks to image classification [14] and speech recognition [15], we combine CNN with the Tree-LSTM 


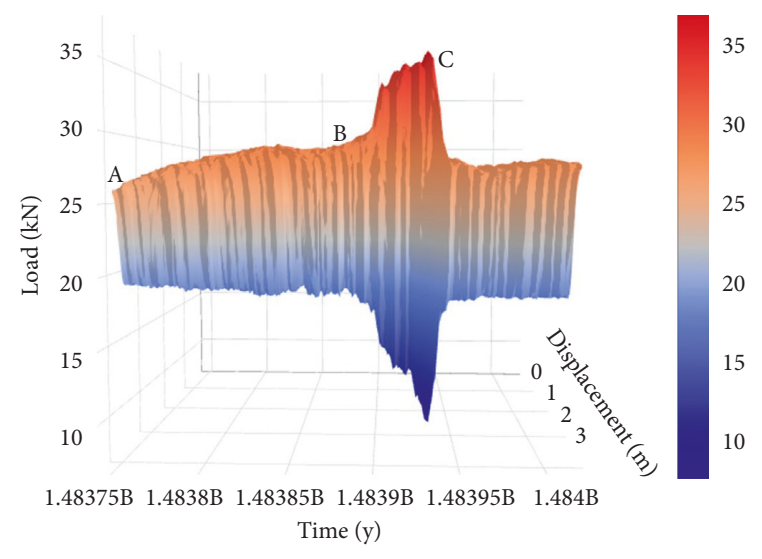

(a)

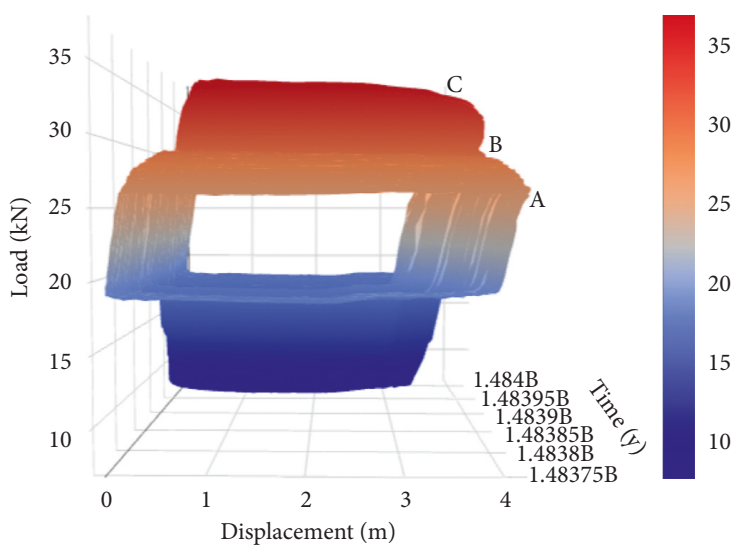

(b)

FIgURE 2: Indicator diagram dataset of scale deposition. (a) View. (b) Side view. $X$-axis represents time, the $Y$-axis represents load, and the $Z$-axis represents displacement.

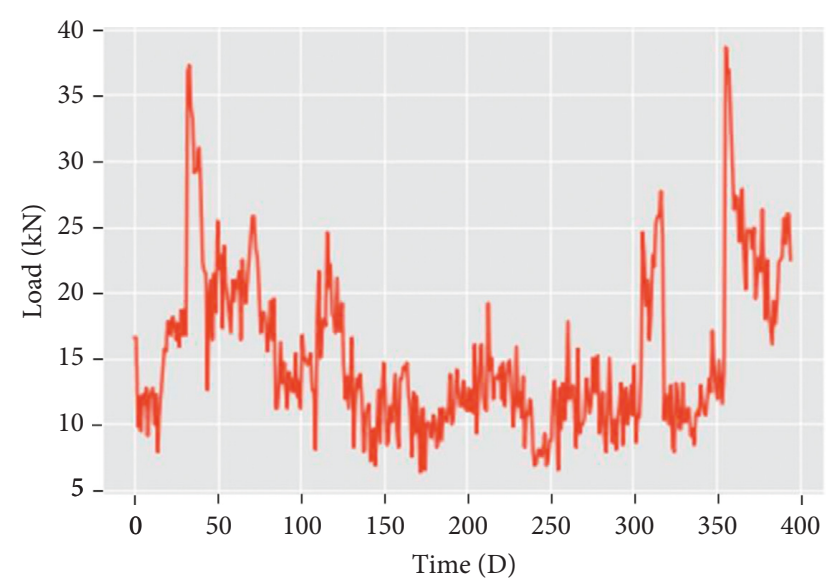

FIgURE 3: Load variation identified by CNN-LSTM.

network, which can not only effectively extract the local features in an indicator diagram but also prevent the gradient disappearance phenomenon to occur in the time axis.

3.4. CNN Structure. CNN contains three types of layers: input, convolution and pooling, and fully connected layers. In the input layer, information received consists of multiple image sequences $\left\{A_{1}, A_{2}, \ldots, A_{n}\right\}$, which are composed of the indicator diagram dataset. In the dataset-collection process, the different collection points are synchronized using the difference-fitting method. The feature parameters are obtained by CNN, which mainly include the suspended point load of the rod pumping system. By analyzing the similarity factor $\left(S_{\mathrm{PCA}}\right)$ of the indicator diagram between the unknown and different typical working conditions, we can identify the types of unknown working conditions and realize workingcondition diagnosis.

In the convolution and pooling layer, we use $n$ convolution filters in each dataset to extract $n$ feature vectors from the indicator diagram and obtain $n$ feature maps, which are also known as $n$ channels. All the feature vectors are stacked in regional matrix $X_{A_{n}}^{\text {map }}$ as follows:

$$
X_{A_{n}}^{\text {dataset }}=\operatorname{ReLU} \text { (dataset, An). }
$$

The structure of the CNN hidden layer is composed of several convolutional and pooling layers. Rectified linear unit $(\mathrm{ReLu})$ is a nonlinear activation function that runs on neurons. The convolution layer contains filter $W_{C} \in R^{d \times h}$, where $h$ is the step size of the filter and $d$ is the dimension of the $n$ feature vectors. The feature vector generated by the filter is expressed as follows [16]:

$$
C=f\left(\operatorname{conv}\left(X_{A_{n}}^{\text {dataset }} * W_{C}\right)+b\right),
$$

where $b$ is a bigoted vector, that is, the intercept of the function that is used to complete an accurate linear classification, as shown in Figure 5.

The pooling layer subsamples the output of the convolutional layer. The most common method to perform pooling is to apply a max operation to the result of each filter and obtain the output value as follows:

$$
X_{A_{n}}^{\mathrm{map}}=[\max \{C\}] .
$$

Two reasons justify the use of the max-pooling operation. First, by eliminating the nonmaximal values, it reduces the computation of the upper layers. Second, it provides a form of translation invariance. The obtained feature vectors are then fed to the sequential layer. To capture the longdistance dependence, LSTM is introduced into the sequential layer for vector composition [3].

3.5. LSTM Structure. LSTM [17] adds a gate to the architecture. Through the combined action of the input, output, and forget gates on the input data, it can solve the problem of vanishing and exploding gradients that exist in RNN [18]. However, ordinary LSTM suffers from many obvious limitations, e.g., it only solves linear problems, it is over dependent on the training sequence, and it is not predictive [19]. Thus, the tree-structure is proposed. Figure 6 shows the LSTM structure. 


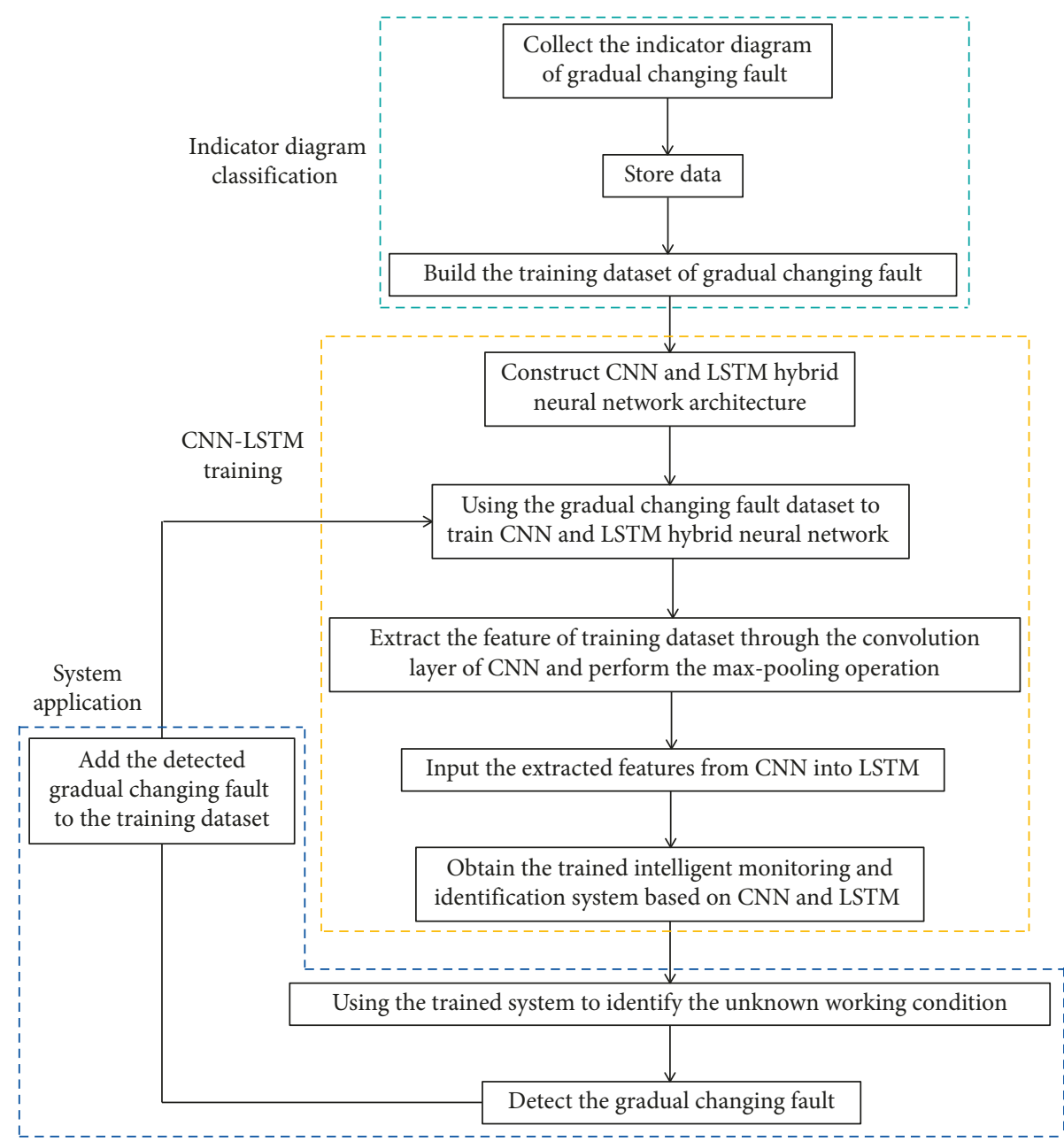

FIGURE 4: Framework overview of the proposed method.
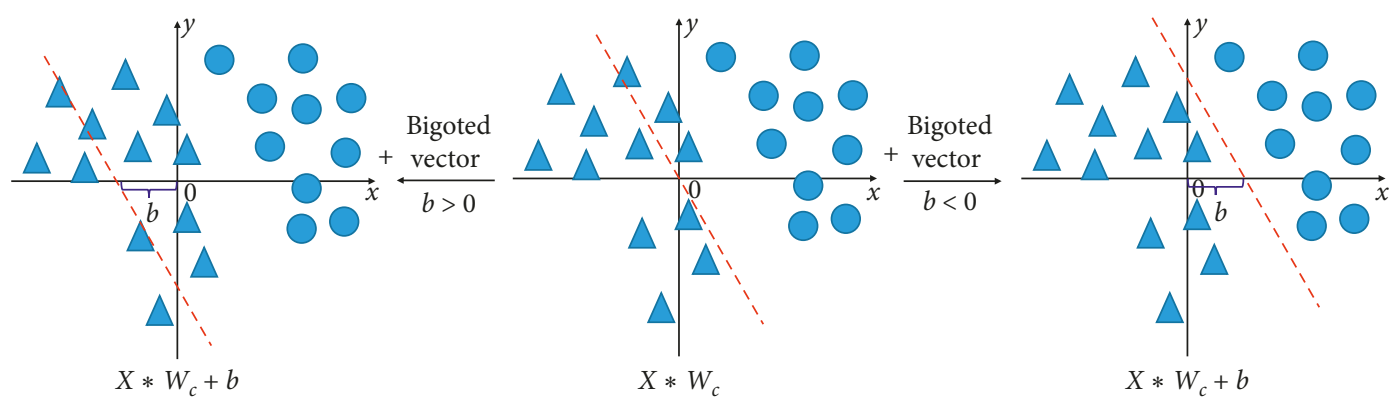

FIGURE 5: "Bigoted vector" performing linear classification.

For each parent node, two child nodes exist, namely, $h_{n}$ and $c_{n}$, which represent the node hidden state and memory unit, respectively. The transfer equation of node $n$ is expressed as follows:

$$
\left[\begin{array}{c}
c_{n} \\
f_{n}^{l} \\
f_{n}^{r}
\end{array}\right]=\left[\begin{array}{c}
\tanh \\
\sigma \\
\sigma
\end{array}\right] T_{a, b}\left[\begin{array}{c}
X_{j} \\
h_{n}^{l} \\
h_{n}^{r}
\end{array}\right],
$$

where $X_{j}$ stands for the input vector, superscripts $l$ and $r$ represent the left and right child nodes, respectively, $\sigma$ is the sigmoid function, and $T_{a, b}$ denotes the linear change, where $a$ and $b$ are the network parameters to be learned.

Then, we connect the output end of LSTM to the softmax classifier and obtain the classification result of the workingcondition recognition [20]. Figure 7 shows the entire proposed CNN-LSTM model. 


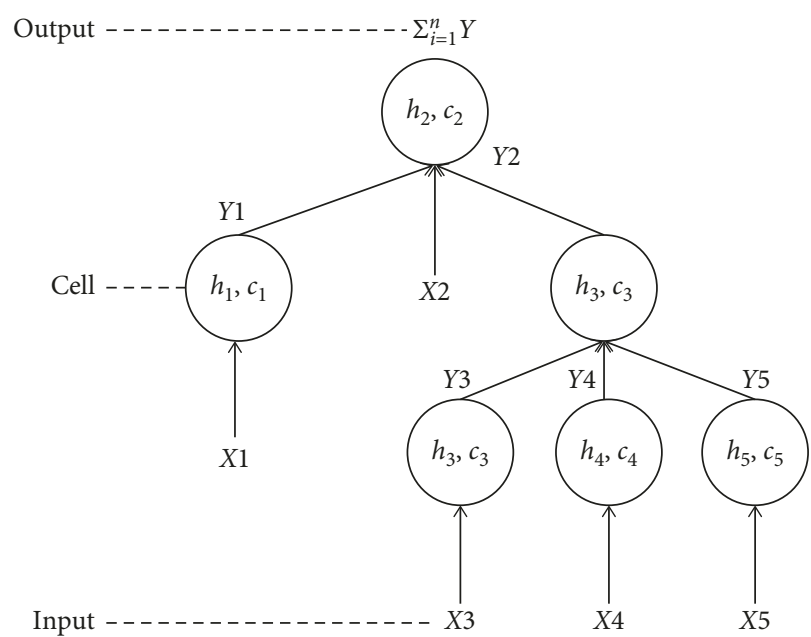

Figure 6: Added tree-structure to LSTM ( $X$ is the input feature data from CNN and $Y$ is the predicted output of LSTM).

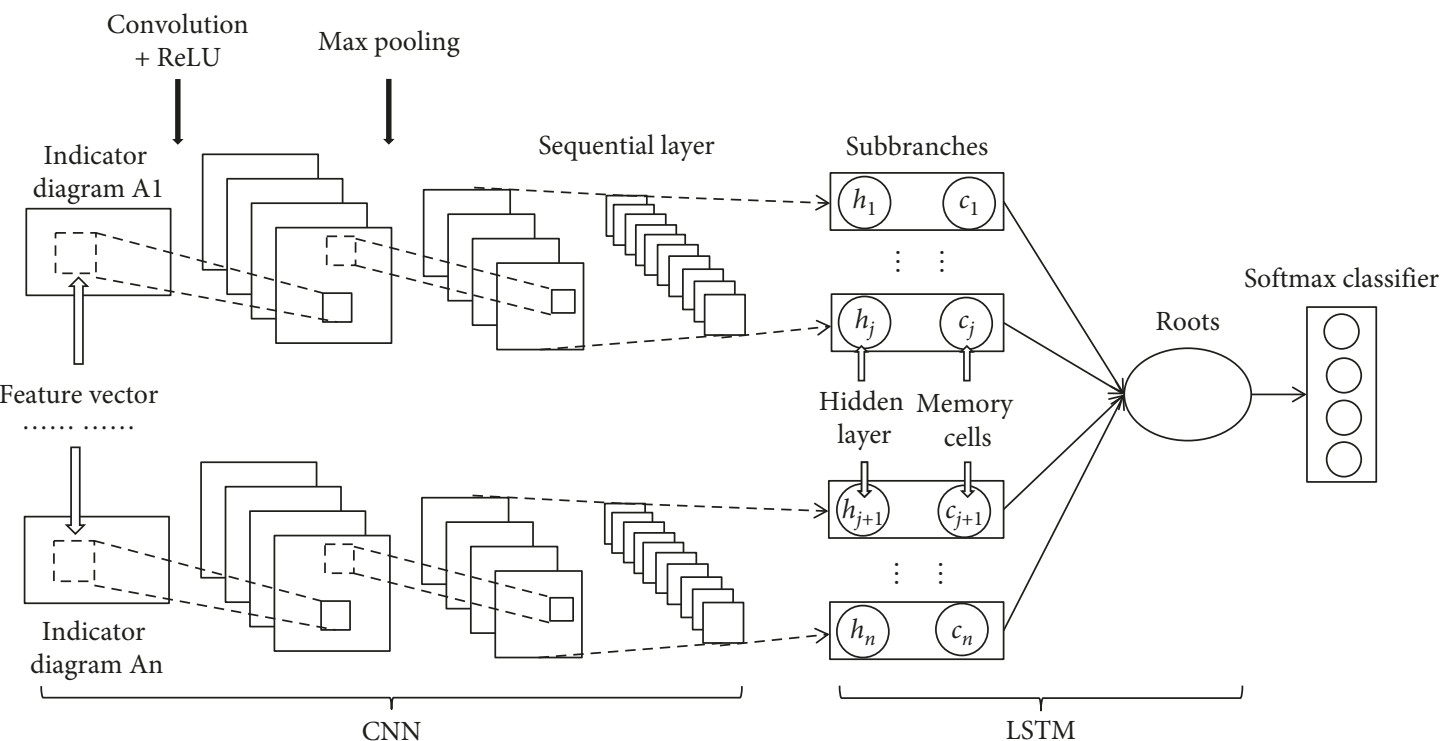

Figure 7: CNN-LSTM model.

\section{Experiments}

In this section, we present our conducted orthogonal experiments to optimize the CNN-LSTM parameters. The CNN-LSTM structure was defined in Section 3. Subsequently, the combination of the neural network parameters with different scales is tested. Finally, the optimal parameter setting mode is determined.

4.1. Setting. CNN-LSTM experiments are conducted on the training dataset consisting of 1.3 million sets of wellproduction test data. The parameter settings are listed in Table 2.

The neural network recognition accuracy of the seventh architecture is shown to be as high as $96.1 \%$. The selected CNN convolution kernel parameters are 16, 32, 64, and 128.
4.2. Evaluation of CNN-LSTM. For the datasets, $80 \%$ of the indicator diagrams are used for training and $20 \%$ are used for testing. The proposed method is evaluated based on the categorization accuracy, which is defined as follows:

accuracy $=\frac{\text { number of correctly categorized indicator diagrams }}{\text { total number of indicator diagrams }}$.

The training result of CNN-LSTM is shown in Figures $8(\mathrm{a})$ and $8(\mathrm{~b})$. The accuracy and loss results are shown in Figure 8(c).

The blue part in Figure 8(a) shows the recognition of the training dataset, and the purple part shows the test dataset recognition. Figure $8(\mathrm{~b})$ shows that the recognition results both in the training and test datasets are superior. The training accuracy reaches $96.7 \%$, whereas the testing accuracy reaches $94.1 \%$. From the recognition results, we can 
TABLe 2: Parameter setting.

\begin{tabular}{lcccccccc}
\hline No. & Convolution layers & Cove & Pooling layers & Sampling rate & Hidden layers & Dropout & Train time (h) & Accuracy (\%) \\
\hline 1 & 2 & $16: 32$ & 2 & $2 \times 2$ & $1 / 136$ & 0 & 183 \\
2 & 4 & $16: 32: 64: 128$ & 4 & $2 \times 2$ & $2 / 2048: 512$ & 0.25 & 276 & 92.1 \\
3 & 2 & $16: 32$ & 2 & $2 \times 2$ & $1 / 136$ & 0 & 159 & 92.5 \\
4 & 4 & $16: 32: 64: 128$ & 4 & $2 \times 2$ & $2 / 2048: 512$ & 0.25 & 264 & 92.6 \\
5 & 2 & $16: 32$ & 2 & $2 \times 2$ & $1 / 136$ & 0 & 76 & 92.9 \\
6 & 2 & $16: 32$ & 2 & $2 \times 2$ & $2 / 2048: 512$ & 0.25 & 83 & 94.2 \\
7 & 4 & $16: 32: 64: 128$ & 4 & $2 \times 2$ & $2 / 2048: 512$ & 0.25 & 135 & 96.1 \\
8 & 4 & $16: 32: 64: 128$ & 4 & $2 \times 2$ & $2 / 2048: 512$ & 0.5 & 168 & 95.7 \\
\hline
\end{tabular}

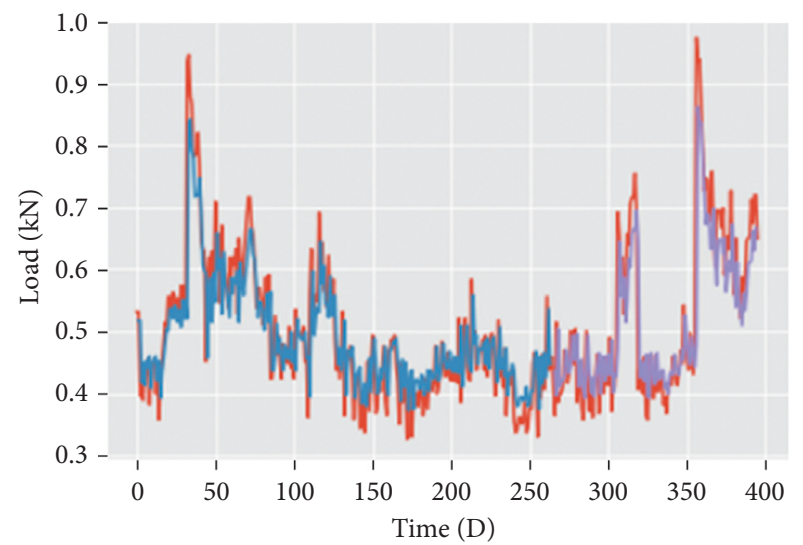

(a)

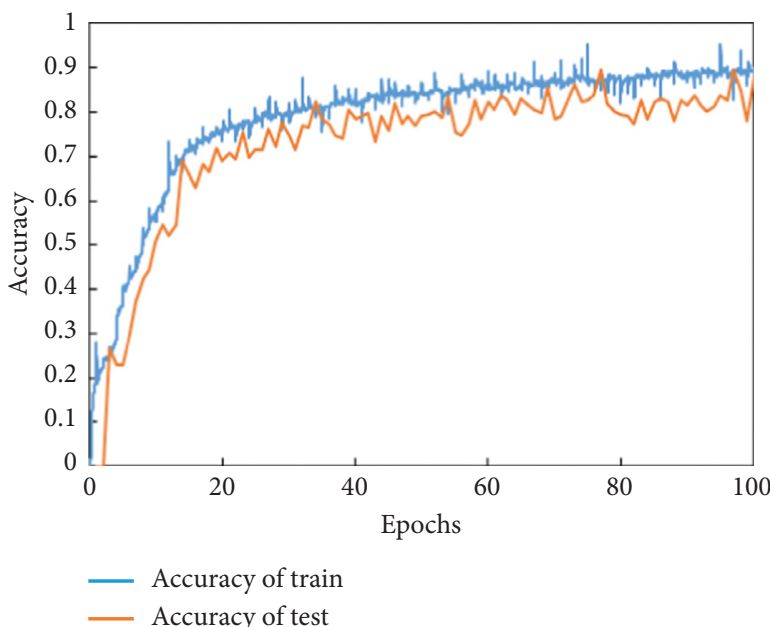

(b)

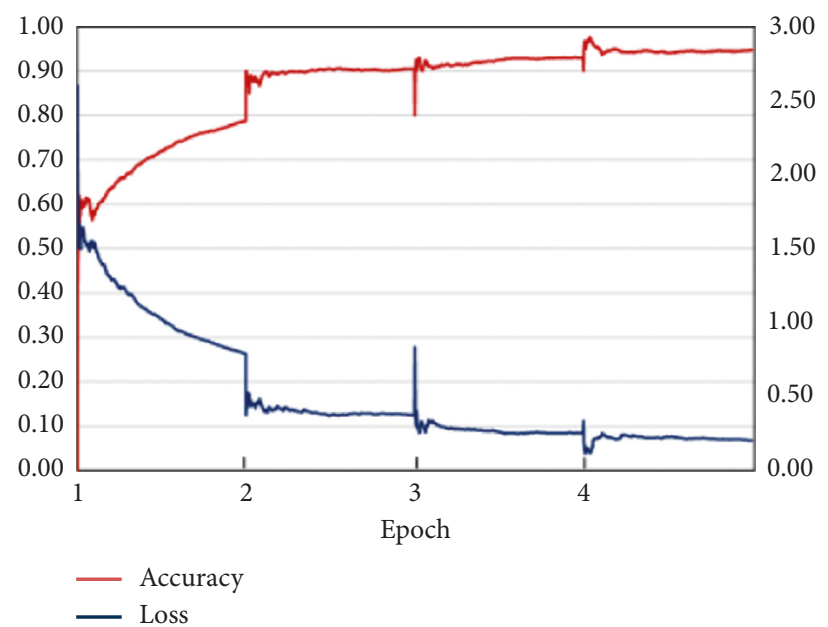

(c)

Figure 8: CNN-LSTM training results. (a) Training and testing dataset recognition. (b) Training and testing accuracy. (c) Accuracy and loss of CNN-LSTM.

observe that the proposed CNN-LSTM is quite robust against changes in the training data. By choosing a sufficient training time, the epoch can yield reasonable and accurate identification results.

Figure $8(\mathrm{c})$ shows that as the training epoch becomes large, the accuracy of the proposed CNN-LSTM identification method accordingly increases, and finally, the accuracy reaches $98.4 \%$. The loss is less than $0.9 \%$. As this epoch increases, CNN-LSTM becomes more powerful, which can process complex gradual changing fault recognition. Here, overfitting is not a problem because it can be easily overcome by early training stop.

4.3. Comparison with Other Methods. At present, the main method of fault recognition is to design experiments to 


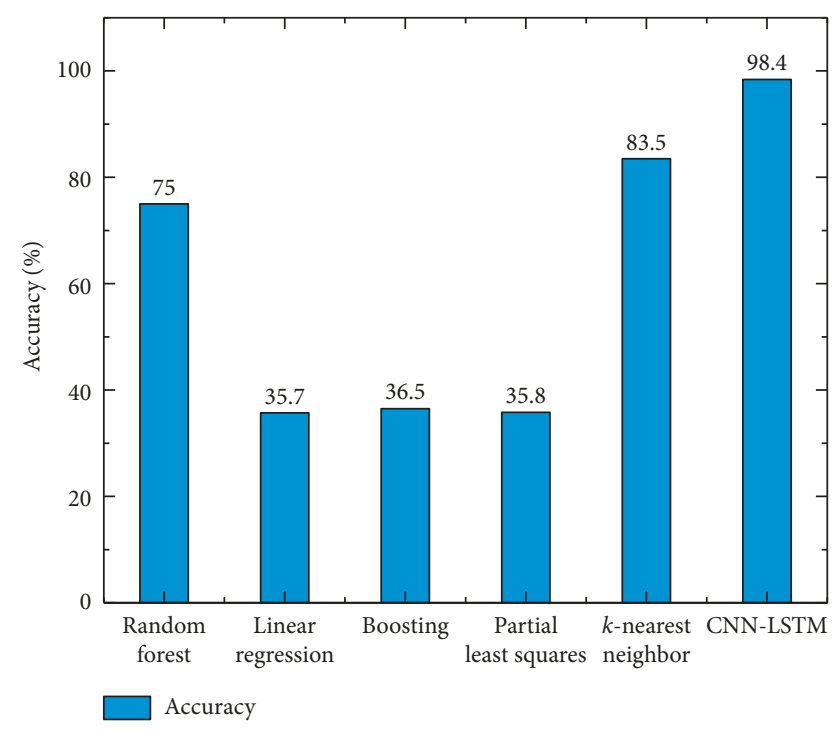

FIGURE 9: Accuracy of the algorithm and CNN-LSTM method.

simulate the working environment of a rod pumping system and then employ the theoretical mathematical model based on the experimental data. Next, different algorithms are adopted to calculate the established model. Fault diagnosis is influenced by the data. According to the literature [21], five analysis algorithms, namely, random forest, linear regression, boosting, partial least square, and K-nearest neighbor algorithms are adopted by the SPSS Model tools. To identify the fault, the accuracy is considered as an evaluation index. The comparison of the traditional and CNN-LSTM methods is shown in Figure 9.

Compared with the other methods, the CNN-LSTM method overcomes the limitations of the theoretical analysis in the traditional models and improves the diagnosis accuracy. The traditional model contains many assumptions, which lead to a large deviation between the actual production environment and theoretical value. Thus, the theoretical model cannot achieve high-precision identification. In addition, the oilfields are widely equipped with automatic data-acquisition equipment to realize real-time monitoring of oil-well status and collect and save production data. The neural network performs better in dealing with a large number of data processing. In addition, the neural network has shown absolute advantages in data mining of hidden values and improves data utilization.

\section{Conclusion}

In this paper, we have proposed the combination of $\mathrm{CNN}$ and LSTM to perform gradual changing fault identification using the indicator diagram. We utilize the hierarchical structure of CNN to create image features from the indicator diagram and employ the recurrent structure of LSTM to integrate the features. We determine the periodic change in a gradual changing fault load. Corresponding to each indicator diagram, a softmax classifier is trained based on the outputs of LSTM, whose outputs are combined to identify the type of input fault. We evaluate the proposed $\mathrm{CNN}$ -
LSTM recognition method using 1.3 million datasets. The experiments demonstrate the effectiveness of utilizing CNN and LSTM to recognize the gradual changing fault using the indicator diagram and characteristic parameters. Experiments on the proposed CNN-LSTM recognition method demonstrate that the accuracy reaches $98.4 \%$, and the loss is less than $0.9 \%$.

\section{Data Availability}

All data and program files included in this study are available upon request by contact with the corresponding author.

\section{Conflicts of Interest}

The authors declare that they have no conflicts of interest.

\section{Authors' Contributions}

Dr. Yanfeng provided a lot of academic guidance for this article; Dr. Yali Liu is mainly responsible for the writing and giving response to the review of this paper; Mr. Shuai Shao has made contributions to the figure and table modification in this paper; Mr. Xuhang Zhao completed the language improvement in the process of manuscript modification; Mr. Guojun Liu made contributions to the collection and arrangement of the production data of oil well; Mr. Xiangji Kong has given lots of advice according to his work experience during the modification of the manuscript; and Ms. $\mathrm{Lu} \mathrm{Liu}$ is responsible for checking and proofreading the final accepted manuscript file.

\section{Acknowledgments}

This study was supported by the Natural Science Research Project of Higher Education of Jiangsu, China (no. 17KJB440001).

\section{References}

[1] A. S. Velichkovich, "Shock absorber for oil-well sucker-rod pumping unit," Chemical \& Petroleum Engineering, vol. 41, no. 9-10, pp. 544-546, 2005.

[2] A. M. F. D. Souza, M. A. D. Bezerra, M. D. A. B. Filho, and L. Schnitman, "Using artificial neural networks for pattern recognition of downhole dynamometer card in oil rod pump system," in Proceedings of the 8th WSEAS international conference on Artificial intelligence, knowledge engineering and data bases AIKED'09, Cambridge, UK, February 2009.

[3] L. O. Chua and T. Roska, "The CNN paradigm," IEEE Transactions on Circuits and Systems I: Fundamental Theory and Applications, vol. 40, no. 3, pp. 147-156, 1993.

[4] H. Ying, K. Li, and H. Huang, "The downhole fault prediction method of rod pumping well based on multivariable gray model," CN201510248922.9, Bohai University, Jinzhou, China, 2016.

[5] J. P. C. Chiu and E. Nichols, "Named entity recognition with bidirectional LSTM-CNNs," Computer Science, vol. 7, 2016.

[6] S. Merity, N. S. Keskar, and R. Socher, "Regularizing and optimizing LSTM language models," 2017, https://arxiv.org/ abs/1708.02182. 
[7] K. S. Tai, R. Socher, and C. D. Manning, "Improved semantic representations from tree-structured long short-term memory networks," Computer Science, vol. 5, no. 1, p. 36, 2015.

[8] E. Kiperwasser and Y. Goldberg, "Easy-first dependency parsing with hierarchical tree LSTMs,” 2016, https://arxiv.org/ abs/1603.00375.

[9] A. Singhal and D. E. Seborg, "Pattern matching in historical batch data using PCA," IEEE Control Systems, vol. 22, no. 5, pp. 53-63, 2002.

[10] T. Kourti and J. F. Macgregor, "Multivariate SPC methods for process and product monitoring," Journal of Quality Technology, vol. 28, no. 4, pp. 409-428, 1996.

[11] W. J. Krzanowski, "Between-group comparison of principal components some sampling results," Journal of Statistical Computation \& Simulation, vol. 15, no. 2-3, pp. 141-154, 1982.

[12] B. J. Gregory, T. L. Beck, R. G. Peterson, M. A. Macdonald, and M. D. Dry, "Rod pump apparatus and method," US8708671B2, Unico, Inc., Franksville, WI, USA, 2009.

[13] X. Ma and E. Hovy, "End-to-end sequence labeling via bidirectional LSTM-CNNs-CRF,” 2016, https://arxiv.org/abs/ 1603.01354 .

[14] A. Krizhevsky, I. Sutskever, and G. Hinton, "ImageNet classification with deep convolutional neural networks," Advances in Neural Information Processing Systems, vol. 25, no. 2, 2012.

[15] O. Abdel-Hamid, A.-R. Mohamed, H. Jiang, L. Deng, G. Penn, and $\mathrm{D}$. Yu, "Convolutional neural networks for speech recognition," IEEE/ACM Transactions on Audio, Speech, and Language Processing, vol. 22, no. 10, pp. 1533-1545, 2014.

[16] S. Ren, K. He, R. Girshick, and J. Sun, "Faster R-CNN: towards real-time object detection with region proposal networks," IEEE Transactions on Pattern Analysis and Machine Intelligence, vol. 39, no. 6, pp. 1137-1149, 2017.

[17] A. Graves and J. Schmidhuber, "Framewise phoneme classification with bidirectional LSTM and other neural network architectures," Neural Networks, vol. 18, no. 5-6, pp. 602-610, 2005.

[18] Y. Lecun, L. Bottou, Y. Bengio, and P. Haffner, "Gradientbased learning applied to document recognition," Proceedings of the IEEE, vol. 86, no. 11, pp. 2278-2324, 1998.

[19] J. Y. Choi and B. Lee, "Combining LSTM network ensemble via adaptive weighting for improved time series forecasting," Mathematical Problems in Engineering, vol. 2018, Article ID 2470171, 8 pages, 2018.

[20] G. Hinton and R. Salakhutdinov, "Discovering binary codes for documents by learning deep generative models," Topics in Cognitive Science, vol. 3, no. 1, pp. 74-91, 2011.

[21] L. C. Briand, W. L. Melo, and J. Wust, "Assessing the applicability of fault-proneness models across object-oriented software projects," IEEE Transactions on Software Engineering, vol. 28, no. 7, pp. 706-720, 2002. 


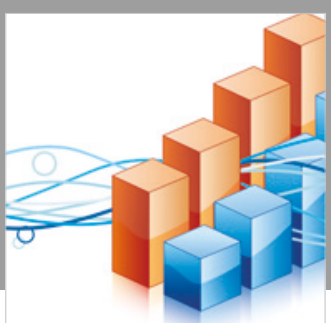

Advances in

Operations Research

\section{-n-m}
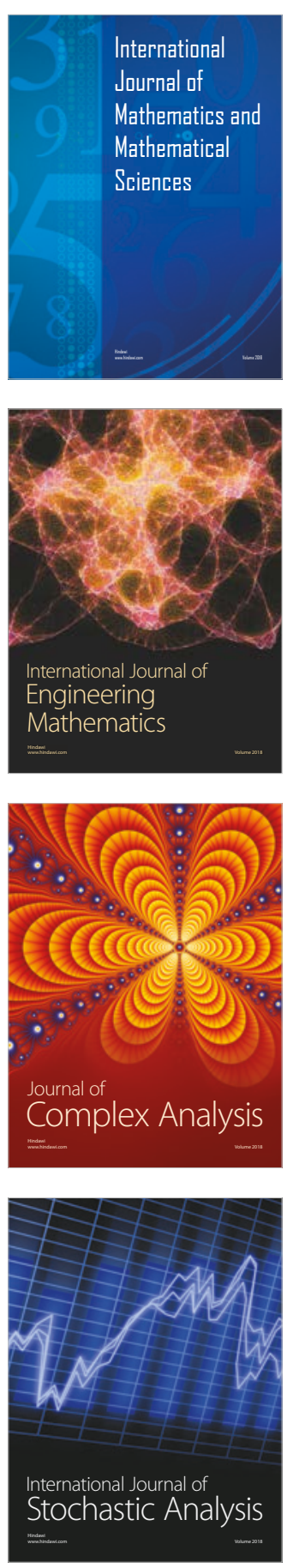
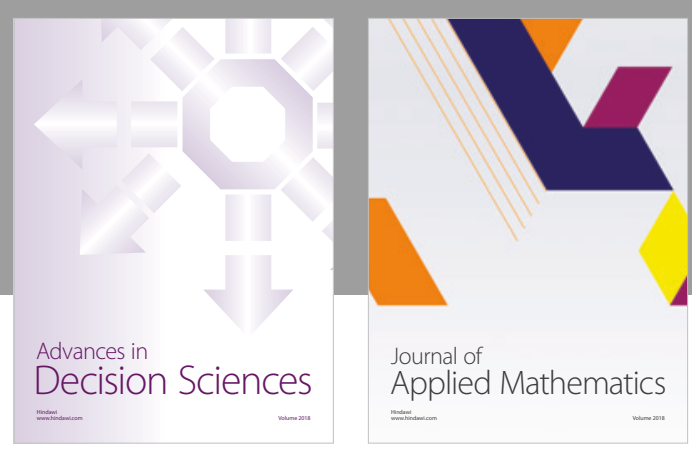

Journal of

Applied Mathematics
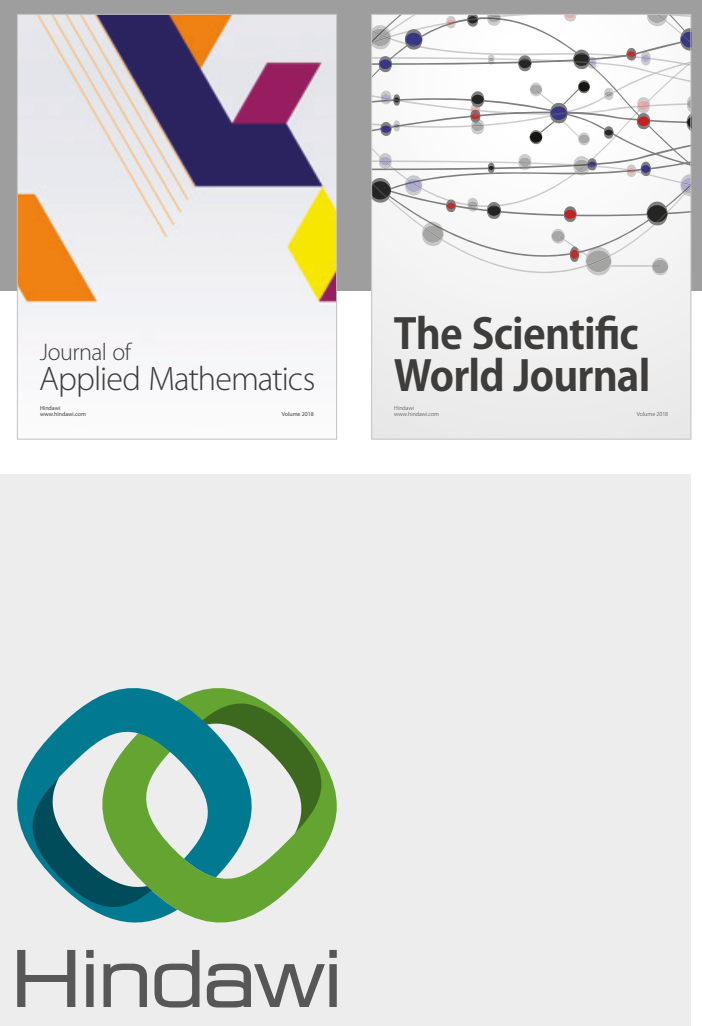

Submit your manuscripts at

www.hindawi.com

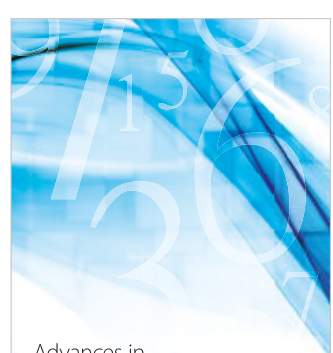

Advances in
Numerical Analysis
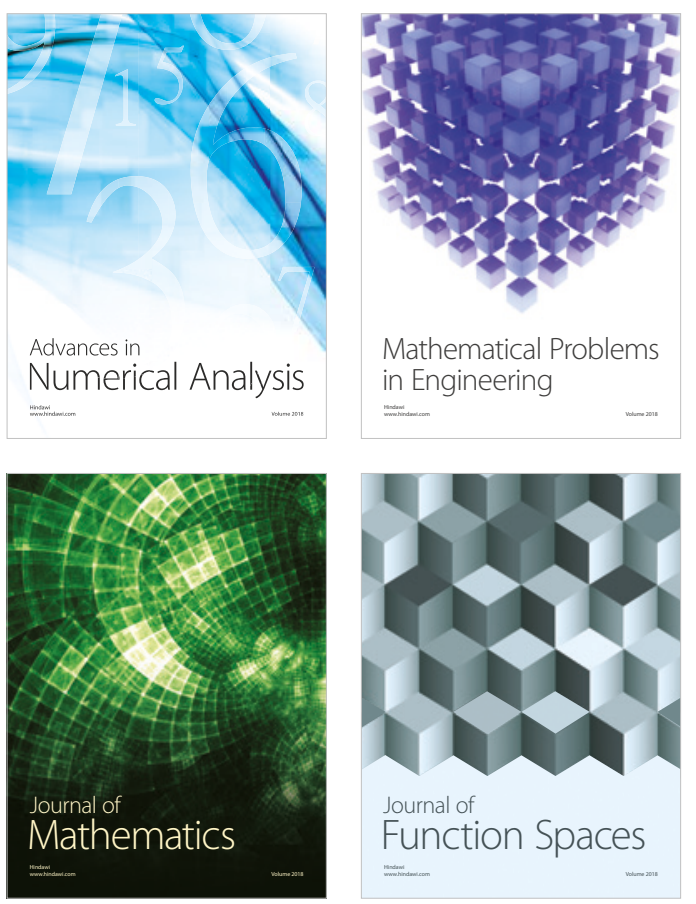

Mathematical Problems in Engineering

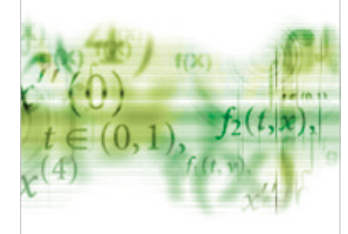

International Journal of

Differential Equations

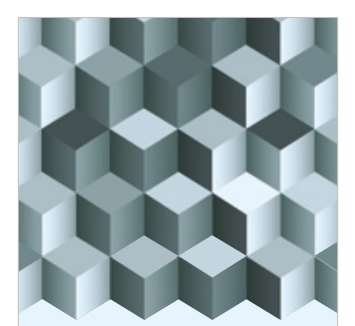

Journal of

Function Spaces
The Scientific

World Journal

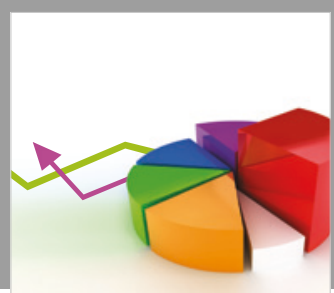

Journal of

Probability and Statistics
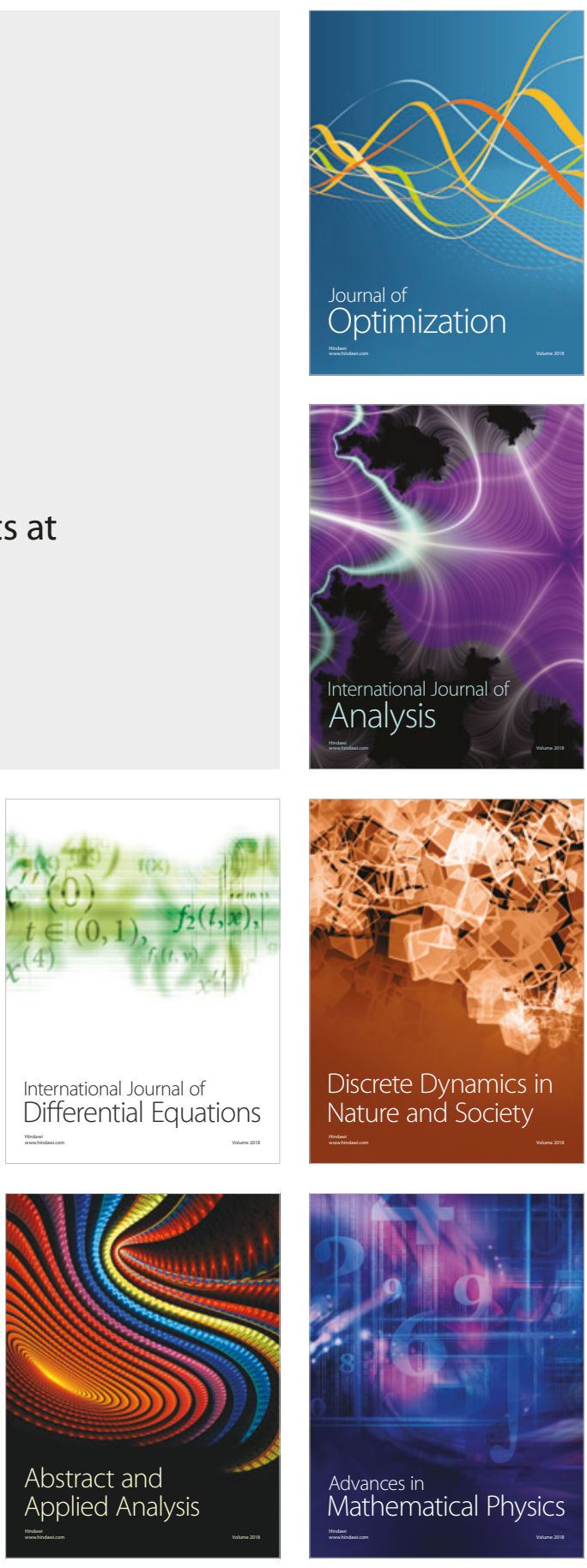